\title{
Instrumentos de Autorregulação Emocional: Uma Revisão de Literatura
}

\author{
Helder Henrique Viana Batista ${ }^{1}$, Ana Paula Porto Noronha \\ Universidade São Francisco, Campinas-SP, Brasil
}

\section{RESUMO}

O presente estudo objetivou analisar a produção científica relativa aos instrumentos que avaliam a autorregulação emocional por meio de uma revisão integrativa de literatura de uma base de dados brasileira, SciELO, no período entre 2008 e 2017 . Foram analisados 19 artigos, considerando os instrumentos mais utilizados, ano e país de publicação, construtos relacionados à regulação das emoções e amostras de aplicação dos instrumentos. O maior número de publicações se deu em 2016 e 2014. A inteligência emocional foi o construto mais estudado e a Trait Meta-Mood Scale o instrumento mais utilizado. A amostra mais frequentemente pesquisada foi a de adultos. Conclui-se que o os modelos do Emotional Regulation Questionnaire e da Difficulties in Emotion Regulation Scale são os mais utilizados para avaliar a autorregulação. Ademais, o número de pesquisas sobre a autorregulação emocional tem aumentado, sendo esta um importante recurso na preservação da saúde.

Palavras-chave: inteligência emocional, ansiedade, autocontrole.

\section{ABSTRACT - Emotional Self-Regulation Instruments: A Literature Review}

The present study aimed to analyze the scientific production related to instruments that evaluate emotional self-regulation through an integrative literature review through a search in the Brazilian SciELO database for the period 2008 to 2017. We analyzed 19 articles, considering the most-used instruments, publication year and country, constructs related to emotional regulation, and instrument application samples. The largest number of publications occurred in 2016 and 2014. Emotional intelligence was the most studied construct and the Trait Meta-Mood Scale was the most widely used instrument. The most frequently searched sample was adults. It is concluded that the models of the Emotional Regulation Questionnaire and the Difficulties in Emotion Regulation Scale are the most used to evaluate self-regulation. In addition, the number of studies on emotional self-regulation has increased, given that this an important resource in the preservation of health.

Keywords: emotional intelligence; anxiety; self-control.

\section{RESUMEN - Instrumentos de Autorregulación Emocional: Una Revisión de Literatura}

El presente estudio objetivó analizar la producción científica relativa a los instrumentos que evalúan la autorregulación emocional por medio de una revisión integrativa de literatura de una base de datos brasileña SciELO, en el período entre 2008 y 2017. Se analizaron 19 artículos, considerando los instrumentos más utilizados, año y país de publicación, constructos relacionados a la regulación de las emociones y muestras de aplicación de los instrumentos. El mayor número de publicaciones se dio en el 2016 y 2014. La inteligencia emocional fue el constructo más estudiado y la Trait Meta-Mood Scale el instrumento más usado. La muestra investigada con más frecuencia fue la de adultos. La conclusión es que los modelos del Emotional Regulation Questionnaire y de la Difficulties in Emotion Regulation Scale son los más utilizados para evaluar la autorregulación. Además, el número de investigaciones sobre la autorregulación emocional ha aumentado, siendo un importante recurso en la preservación de la salud.

Palabras clave: inteligencia emocional; ansiedad; autocontrol.

Autorregulação emocional ou regulação emocional refere-se a um processo dinâmico intrinsecamente ligado a esforços conscientes no controle dos comportamentos, dos sentimentos e das emoções para que algum objetivo seja alcançado (Gratz \& Roemer, 2004; McKnow, Gumbiner, Russo, \& Lipton, 2009; Santana \& Gondim, 2016). O controle das próprias emoções se desenvolve gradualmente na infância e na adolescência e é um importante recurso para as exigências de adaptação inerentes à vida humana, no contexto educativo ou no organizacional, na ingestão de alimentos e substâncias e nos relacionamentos interpessoais (Barros, Goes, \& Pereira, 2015; Berking, Wirtz, Svaldi, \& Hofmann, 2014; Castillo, Dias, \& Castelar-Perim, 2012; Cruvinel 
\& Boruchovitch, 2011; Sheppes, Scheibe, Suri, \& Gross, 2011).

Duas propriedades básicas da autorregulação têm sido consideradas. A primeira se refere ao sistema motivacional e dinâmico de estabelecimento, desenvolvimento e implementação de objetivos; análise dos progressos realizados e revisão das metas e estratégias determinadas anteriormente. Por sua vez, a segunda propriedade da autorregulação está ligada ao controle das respostas emocionais (Gaspar, Tomé, Simões, \& Matos, 2015).

Em relação ao segundo elemento, o controle das próprias emoções constitui-se em um aspecto protetivo para as pessoas, uma vez que auxilia no controle da impulsividade. A relação entre a impulsividade e o controle emocional é inversa, ou seja, quanto maior o controle emocional, menor a impulsividade (Tharp, Schumacher, McLeish, Samper, \& Coffey, 2012). Além disso, o controle emocional é utilizado como uma forma de tratamento para indivíduos com transtornos de personalidade, sobretudo o Transtorno Borderline ou em terapias para usuários de substâncias psicoativas (Amorim Neto \& True, 2011; Tharp et al., 2012). Controlar as próprias emoções torna-se importante em razão dos comportamentos impulsivos serem características marcantes em alterações neuropsicológicas de idosos, ou ainda no Transtorno de Déficit de Atenção/Hiperatividade (Lopes, Nascimento, \& Bandeira, 2005; Schlindwein-Zanini, 2010).

Em acréscimo, as estratégias de regulação das emoções são consideradas essenciais, uma vez que minimizam o desenvolvimento de comportamentos problemáticos e auxiliam no desenvolvimento saudável e harmonioso das pessoas no contexto familiar, amoroso, de amizade e no trabalho (Gondim \& Borges-Andrade, 2009). Ademais, é uma habilidade importante para prevenir e diminuir estados depressivos e de ansiedade e comportamento agressivos (Berking Wirtz, Svaldi \& Hofmann, 2014; Gratz \& Roemer, 2004; Gross \& John, 2003).

A autorregulação emocional tem sido considerada um componente da inteligência emocional, que por sua vez diz respeito às habilidades que as pessoas possuem de identificar, nomear e agir em relação aos próprios sentimentos e aos de outras pessoas (Goleman, 2006). Foram desenvolvidos estudos que investigam a relação entre os dois construtos em diversos contextos, tais como clínico, hospitalar, esportivo e acadêmico (EspinozaVenegas, Sanhueza-Alvarado, Ramírez-Elizondo, \& Sáez-Carrillo, 2015; Isaza-Zapata \& Calle-Piedrahitta, 2016; Ruiz, Salazar, \& Caballo, 2012; Teques, LlorcaRamón, Bueno-Carrera, Pais-Ribeiro, \& Teques, 2015). Além de se relacionar com a inteligência emocional, associações com suporte social e estilos parentais também vêm sendo pesquisadas (Gaspar \& Matos, 2015; Pinto, Carvalho, \& Sá, 2014).

A autorregulação emocional tem sido avaliada predominantemente por meio dos modelos conceituais adotados no desenvolvimento da Difficulties in Emotion
Regulation Scale (DERS, de Gratz \& Roemer, 2004) e do Emotional Regulation Questionnaire (ERQ, de Gross \& John, 2003), de forma que instrumentos foram construídos e adaptados em vários países (Cabello, José M., Fernández-Berrocal, \& Gross, 2012; Muñoz-Martínez, Vargas, \& Hoyos-González, 2016; Porro, Andrés, \& Rodrígues-Espínola, 2012). A DERS investiga as dificuldades de regulação das próprias emoções (desregulação emocional). Os quatro fatores possuem itens que identificam: (a) capacidade de tomar consciência e compreender as emoções; (b) aceitar as próprias emoções; (c) controle dos comportamentos impulsivos de modo a se guiar para que determinados objetivos sejam alcançados; (d) acesso a estratégias de autorregulação emocional percebidas como efetivas. A ausência de qualquer uma dessas habilidades poderia indicar dificuldades na regulação das emoções (Gratz \& Roemer, 2004). Por sua vez, o modelo desenvolvido por Gross e John (2003) possui dois fatores que se aplicam a emoções negativas e positivas e que indicam controle das emoções, de forma que os pensamentos são modificados diante da situação vivenciada (estratégias de reavaliação das emoções) e o controle emocional no qual o indivíduo não expressa algumas emoções (supressão das emoções).

O presente estudo teve por objetivo analisar a produção científica acerca da autorregulação emocional entre 2008 e 2017 em uma base de dados brasileira (Scientific Electronic Library Online - SciELO), identificando os instrumentos que avaliam o construto e as propriedades psicométricas dos instrumentos. As revisões de literatura existentes no Brasil abordam a regulação das emoções em focos variados, como a neurobiologia das emoções, os benefícios da regulação emocional em relacionamentos de pais e filhos e em tratamentos de obesidade (Arcoverde \& Soares, 2012; Barros, Goes \& Pereira, 2015; Silva, 2015). Todavia, não foram encontradas revisões que enfatizassem os instrumentos utilizados em trabalhos publicados em bases de dados brasileiras para avaliação do construto. Ter instrumentos para identificar a autorregulação emocional de um indivíduo torna-se um recurso para compreender a origem de problemas psicológicos e, em consequência, para definir o tratamento mais adequado (Nelis, Quoidbach, Hansenne, \& Mikolajczak, 2011), razões pelas quais o estudo é relevante.

\section{Método}

O estudo foi realizado na base de dados brasileira Scientific Electronic Library Online - SciELO, por incluir a maior parte do escopo de publicações científicas do país, para o levantamento da produção científica relacionada aos instrumentos utilizados para avaliação da autorregulação emocional no período entre 2008 e 2017. Como indicado anteriormente, é comum o uso das nomenclaturas "autorregulação emocional" e "regulação emocional" para indicar o controle que as pessoas têm de suas próprias 
emoções. Dessa forma, foram utilizados os dois descritores. O terceiro descritor "regulação emocional e inteligência emocional" foi utilizado, considerando que o primeiro é um componente do segundo (Salovey, Mayer, Goldman, Turvey, \& Palfai, 1995) e que, portanto, poderia contribuir com a recuperação de artigos. Por fim, utilizou-se o descritor "regulação emocional e instrumentos".

Após essa etapa, realizou-se a leitura de resumos $\mathrm{e}$, nos casos em que estes não permitiam ter clareza da metodologia das pesquisas, recorreu-se ao texto integral, sendo incluídas para análise as pesquisas de interesse ao presente estudo, conforme o tema de enfoque e que constassem de medidas que avaliassem a autorregulação emocional. Também foram considerados os trabalhos que incluíssem instrumentos de avaliação de outros construtos com algum fator/dimensão que avaliasse a autorregulação emocional. Foram excluídos artigos duplicados; revisões de literatura; estudos de caso ou estudos com foco em outras áreas de conhecimento, como a medicina, física e ciências sociais; pesquisas que não se referiam ao aspecto psicológico do controle das emoções; estudos nos quais não foi possível identificar, nos instrumentos que avaliassem a regulação emocional, a nomenclatura dos fatores/dimensões, suas definições conceituais e o número de itens, a forma de resposta do instrumento (escalas Likerts ou pranchas, por exemplo) e o alfa de Cronbach. Buscou-se identificar o país e o ano das publicações, os instrumentos utilizados para avaliar a regulação das emoções, quais os construtos que a ela se relacionavam e a amostra a qual os instrumentos foram aplicados.

\section{Resultados}

$\mathrm{Na}$ busca com o descritor "autorregulação emocional", foram encontrados sete artigos relacionados ao tema. Como o número de trabalhos encontrados foi pequeno, utilizou-se o descritor "regulação emocional" e foram encontrados 76 artigos relacionados ao tema, inclusive cinco do primeiro descritor. Nos termos "regulação emocional e inteligência emocional", foram encontrados oito artigos, todos elencados no descritor "regulação emocional". Em "regulação emocional e instrumentos", foram encontrados cinco artigos, três que apareceram anteriormente e dois que não se enquadravam no objetivo do presente estudo, conforme apresentado no fluxograma da Figura 1.

Considerando os critérios de exclusão e inclusão, foram analisados 19 artigos. Inicialmente foram identificados os anos e países dos periódicos de publicação dos artigos. A maioria dos artigos foi publicada no ano de 2016 ( $n=4 ; 21,05 \%)$, seguido do ano de 2014 (15,79\%) com três publicações, 2017, 2015, 2013, 2012 e 2011 com duas publicações em cada ano (10,53\%) e 2010 e 2009 com uma publicação em cada ano $(5,26 \%)$. A maioria dos artigos foi publicada no Brasil ( $n=10 ; 48,15 \%)$, seguido de Colômbia $(n=4 ; 25,93 \%)$, Espanha $(n=3 ; 10,53 \%)$ e Portugal $(n=2 ; 10,53 \%)$.

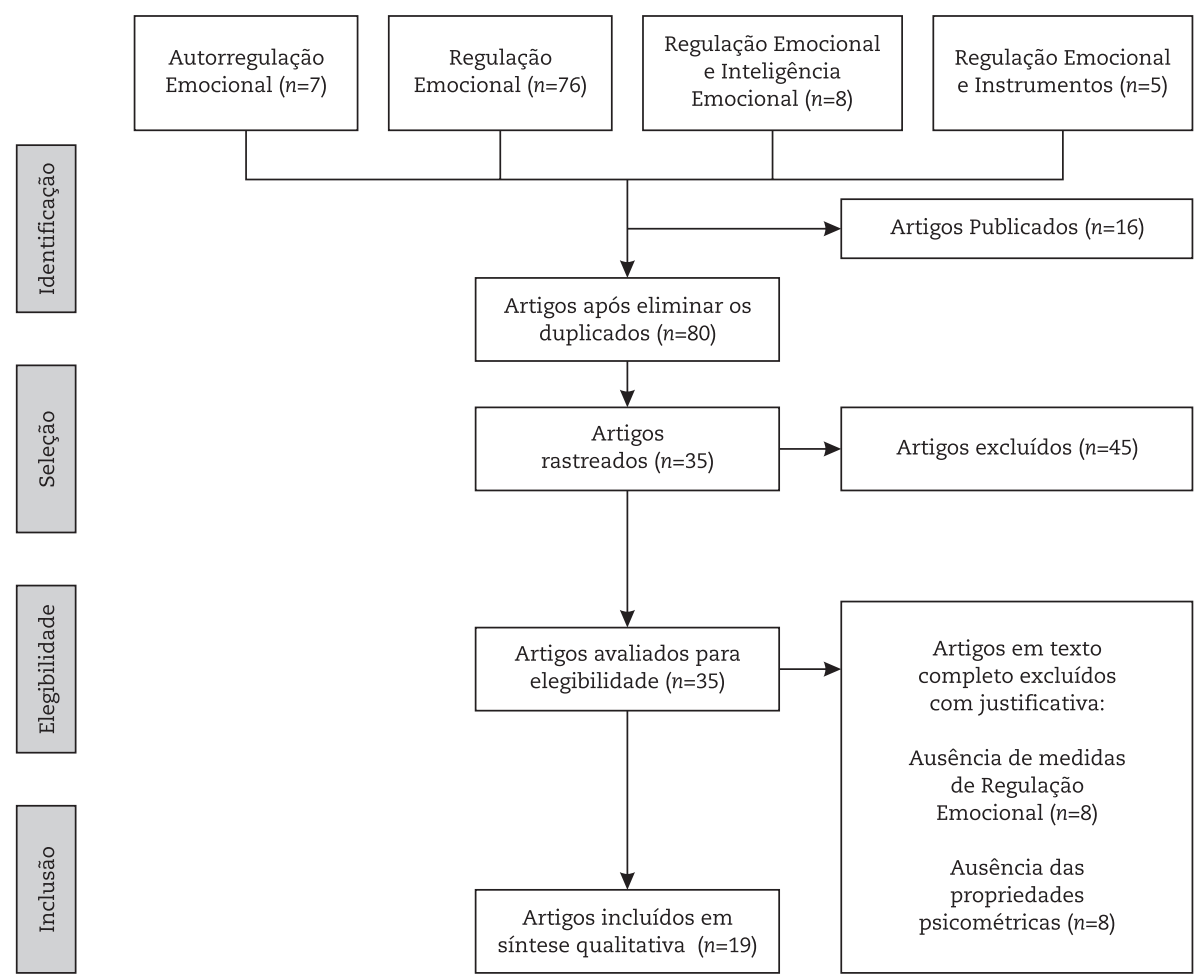

Figura 1. Fluxograma de decisões 
A análise do conteúdo dos artigos permitiu identificar uma variedade de temas relacionados à autorregulação das emoções. O tema que apareceu com maior frequência nos estudos analisados foi "inteligência emocional", presente em sete estudos. Além disso, das temáticas mais frequentes, foi possível identificar estudos que abordavam aspectos relacionados à depressão, ao bem-estar subjetivo e psicológico e ao estresse (três estudos cada). Os temas "afetos", "ansiedade", "atitudes diante da morte", "estresse" e "satisfação com a vida" foram encontrados em dois estudos cada. Por fim, autoeficácia, autoestima, estilos parentais, estratégias de aprendizagem, expressividade emocional, habilidades sociais e personalidade foram temas encontrados em um estudo cada. Vale destacar que dois dos estudos encontrados, tiveram por objetivo construir e validar instrumentos, além de um estudo cujo foco foi desenvolver um programa de intervenção.

No que diz respeito aos instrumentos de avaliação utilizados nas pesquisas, a Trait Meta-Mood Scale (TMMS-24, de Salovey et al., 1995), que avalia a inteligência emocional, foi aplicada na versão espanhola em cinco dos sete estudos que investigavam as relações entre a inteligência emocional e a autorregulação emocional. Os resultados são apresentados na Figura 2.

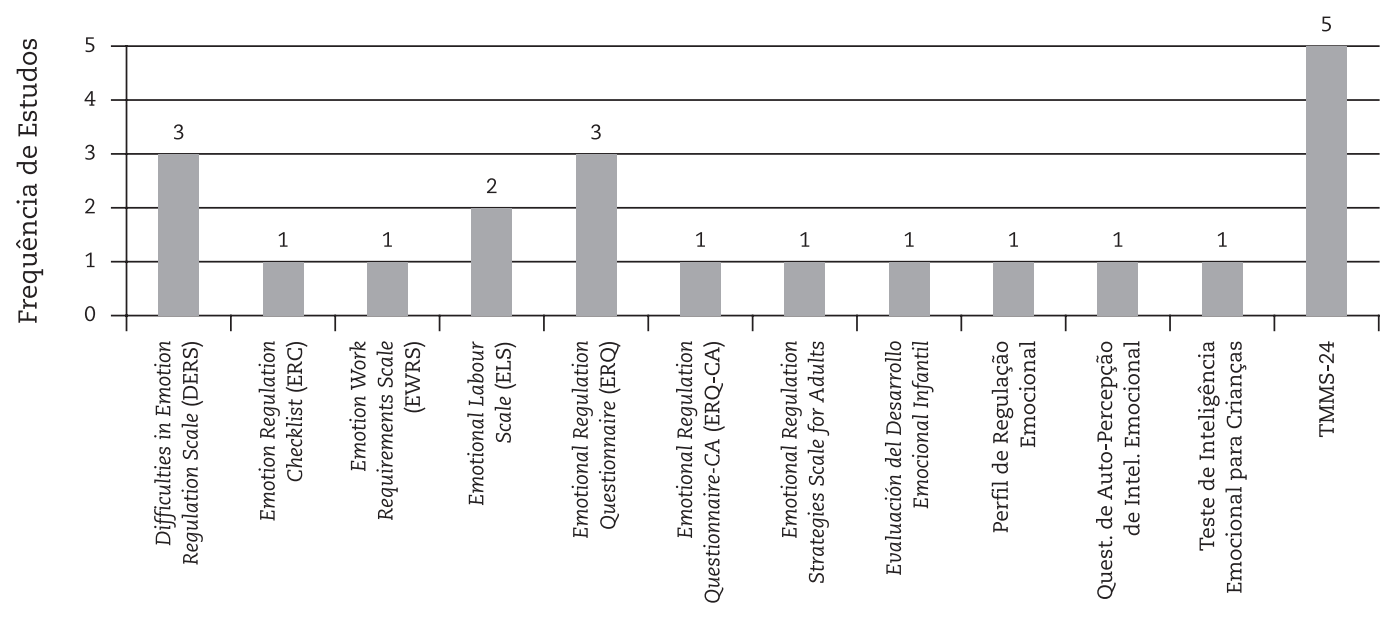

Instrumentos Utilizados

Figura 2. Instrumentos utilizados para avaliar a autorregulação emocional

A TMMS-24 é respondida em uma escala Likert de cinco pontos, sendo originalmente composta por 48 itens e três fatores, com confiabilidades entre 0,82 e 0,87 . $\mathrm{Na}$ versão espanhola, foi encontrada a mesma estrutura fatorial, porém, com 24 itens e confiabilidade entre 0,79 e 086. O fator que se refere ao controle das emoções é reparação $(\alpha=0,86)$, definido como as crenças que o respondente tem acerca de sua capacidade de regular os próprios sentimentos. Além disso, foram encontrados dois instrumentos, Questionário de Autopercepção de Inteligência Emocional (QIE-P, Teques et al., 2015) e o Teste de Inteligência Emocional para Crianças (TIEC, Bueno, 2008), desenvolvidos com base na mesma teoria utilizada na TMMS-24. Os dois instrumentos foram utilizados em um estudo cada. Na Tabela 1, constam as propriedades psicométricas dos instrumentos que avaliavam outros construtos e que possuíam algum fator/dimensão que investigava a regulação emocional encontrados na presente revisão. Foram inseridos na Tabela 1 somente os fatores referentes à regulação emocional.

Depois da TMMS-24, os instrumentos utilizados com maior frequência foram a Emotional Regulation
Questionnaire (ERQ, de Gross \& John, 2003), em três estudos, e a Difficulties in Emotion Regulation Scale (DERS, de Gratz \& Roemer, 2004), em dois estudos. Em relação ao ERQ, tanto a adaptação do instrumento para o Brasil quanto a versão espanhola apresentaram a mesma estrutura, com dois fatores e 10 itens, com alfas de Cronbach entre 0,63 e 0,75 . Cabe ressaltar que a versão espanhola foi utilizada em dois desses estudos, sendo que em um não foi utilizada a reavaliação emocional e em outro não foi informado o alfa de Cronbach desse fator (Granada, Andreu, \& Guiu, 2014; Porro et al., 2012). Além disso, em um estudo, a base teórica utilizada no ERQ também foi utilizada para o desenvolvimento da versão do instrumento para crianças e adolescentes (ERQ-CA, Gullone \& Taffe, 2012), com o mesmo número de itens e fatores e alfas de 0,62 (supressão) e 0,71 (reavaliação).

Quanto à DERS, para responder ao instrumento o participante considera a frase "quando estou triste" $\mathrm{e}$ indica o quanto os comportamentos e emoções o descrevem. A escala foi adaptada para a versão portuguesa, que possui estrutura igual a versão original, com 36 itens, seis fatores e alfas entre 0,75 e 0,88 . Na adaptação para a 
versão brasileira, foi encontrada uma estrutura com 19 itens, três fatores e valores de alfa entre 0,68 e 0,88 e, na versão colombiana, 16 itens, dois fatores e valores de alfas não indicados. Cabe ressaltar que os fatores encontrados nas três versões adaptadas possuem nomenclaturas e definições similares às de Gratz e Roemer (2004).

Ainda, instrumentos como a Emotion Labor Scale (ELS, Brotheridge \& Lee, 2003) e a subescala de Regulação de Emoções da ELS e a Emotion Work Requirements Scale (EWRS, Best, Downey, \& Jones, 1997), ambas utilizadas em um estudo cada, buscam avaliar a regulação emocional no contexto do trabalho. A ELS é uma escala que avalia a superficialidade, a profundidade, a frequência, a duração e a intensidade das emoções no contexto de trabalho em quatro fatores (regulação emocional, variabilidade, intensidade e frequência). Por sua vez, a EWRS busca avaliar a extensão com que se exige cotidianamente do trabalhador o ocultamento das emoções negativas e a expressão das emoções positivas. Ademais, foram encontradas pesquisas que avaliaram a regulação emocional com cenários que estimulavam emoções, como o Perfil de Regulação Emocional - versão reduzida (de Gondim et al., 2015). Os demais instrumentos encontrados e suas propriedades psicométricas estão sumariamente descritos na Tabela 2.

Tabela 1

Instrumentos de Outros Construtos com Algum Fator/Dimensão que Avalie a Regulação das Emoções

\begin{tabular}{|c|c|c|c|}
\hline Instrumentos & $\begin{array}{l}\text { Nomenclatura } \\
\text { das dimensões/ } \\
\text { fatores de regulação } \\
\text { emocional } \\
\left(\mathrm{n}^{\circ} \text { de itens }\right)\end{array}$ & $\begin{array}{c}\text { Definição das dimensões/ } \\
\text { fatores } \\
(\alpha)\end{array}$ & Forma de Resposta \\
\hline $\begin{array}{c}\text { TMMS-24- } \\
\text { Espanha }\end{array}$ & Reparação (8) & $\begin{array}{l}\text { Crença dos indivíduos } \\
\text { sobre sua capacidade } \\
\text { de regular os próprios } \\
\text { sentimentos. }(0,86)\end{array}$ & $\begin{array}{c}\text { Escala Likert de } 5 \text { pontos, sendo } \\
\text { 1="Discordo fortemente" e } \\
\text { 5="Concordo fortemente". }\end{array}$ \\
\hline QIE-P & $\begin{array}{l}\text { Regulação } \\
\text { emocional (3) }\end{array}$ & $\begin{array}{c}\text { Capacidade de controlar } \\
\text { a postura, expressão e } \\
\text { decisão comportamental } \\
\text { perante emoções } \\
\text { negativas ou } \\
\text { positivas. }(0,77)\end{array}$ & Idem ao TMMS-24. \\
\hline TIEC & $\begin{array}{l}\text { Regulação } \\
\text { emocional (8) }\end{array}$ & $\begin{array}{l}\text { Capacidade de identificar } \\
\text { estratégias de coping } \\
\text { apropriadas para } \\
\text { a resolução de } \\
\text { problemas. }(0,57)\end{array}$ & $\begin{array}{l}\text { Composto por tarefas de desempenho } \\
\text { que se referem a oito histórias que } \\
\text { retratam as emoções. As histórias são } \\
\text { apresentadas às crianças no decorrer do } \\
\text { procedimento, utilizando-se um } \\
\text { áudio previamente gravado e a } \\
\text { apresentação de figuras ilustrativas. } \\
\text { A pontuação é dicotômica } \\
\text { (0 para erro e } 1 \text { para acerto). }\end{array}$ \\
\hline $\begin{array}{l}\text { Evaluación } \\
\text { del Desarrollo } \\
\text { Emocional } \\
\text { Infantil }\end{array}$ & $\begin{array}{l}\text { Regulação } \\
\text { emocional (6) }\end{array}$ & $\begin{array}{l}\text { Capacidade de reconhecer } \\
\text { as próprias emoções e } \\
\text { reagir de forma } \\
\text { adequada conforme } \\
\text { a situação. }(0,77)\end{array}$ & $\begin{array}{l}\text { Composto por } 13 \text { lâminas que são } \\
\text { apresentadas aos participantes pelo } \\
\text { avaliador, de forma que este faz } \\
\text { perguntas sobre o conteúdo } \\
\text { das lâminas. }\end{array}$ \\
\hline
\end{tabular}

Tabela 2

Instrumentos Encontrados para Avaliar a Regulação das Emoções

\begin{tabular}{|c|c|c|c|}
\hline Instrumentos & $\begin{array}{l}\text { Nomenclatura das } \\
\text { dimensões/fatores } \\
\left(\mathrm{n}^{\circ} \text { de itens }\right)\end{array}$ & Definição das dimensões/fatores $(\alpha)$ & Forma de Resposta \\
\hline \multirow{3}{*}{ DERS-Brasil } & $\begin{array}{l}\text { Falta de estratégias } \\
\text { de regulação } \\
\text { emocional (9) }\end{array}$ & $\begin{array}{l}\text { Crença de que há pouco a ser feito } \\
\text { para regular as próprias emoções } \\
\text { eficazmente ao vivenciar } \\
\text { emoções negativas. }(0,88)\end{array}$ & \multirow{3}{*}{$\begin{array}{c}\text { Escala Likert de } 5 \\
\text { pontos (1=“quase nunca" } \\
\text { e } 5=\text { "quase sempre"). } \\
\text { Respondente considera a } \\
\text { afirmativa "Quando estou } \\
\text { triste..." para indicar o } \\
\text { que melhor } \\
\text { lhe descreve. }\end{array}$} \\
\hline & $\begin{array}{l}\text { Consciência } \\
\text { emocional (5) }\end{array}$ & $\begin{array}{l}\text { Reflete a tendência a atender } \\
\text { e reconhecer as próprias emoções. }(0,68)\end{array}$ & \\
\hline & $\begin{array}{l}\text { Dificuldade } \\
\text { em controle } \\
\text { de impulso (5) }\end{array}$ & $\begin{array}{c}\text { Capacidade de controlar comportamentos } \\
\text { quando experimenta emoções negativas. } \\
(0,82)\end{array}$ & \\
\hline
\end{tabular}


Tabela 2 (continuação)

Instrumentos Encontrados para Avaliar a Regulação das Emoções

\begin{tabular}{|c|c|c|c|}
\hline Instrumentos & $\begin{array}{l}\text { Nomenclatura das } \\
\text { dimensões/fatores } \\
\left(\mathrm{n}^{\circ} \text { de itens }\right)\end{array}$ & Definição das dimensões/fatores $(\alpha)$ & Forma de Resposta \\
\hline \multirow{6}{*}{ DERS-Portugal } & Estratégias (8) & $\begin{array}{c}\text { Crença de que há pouco a ser feito para } \\
\text { regular as próprias emoções eficazmente } \\
\text { quando está chateado. }(0,88)\end{array}$ & \multirow{6}{*}{$\begin{array}{l}\text { Idem ao } \\
\text { DERS-Brasil }\end{array}$} \\
\hline & Não aceitação (6) & $\begin{array}{c}\text { Tendência a ter respostas emocionais } \\
\text { secundárias negativas, ou não aceitar } \\
\text { as próprias emoções diante } \\
\text { o sofrimento de alguém. }(0,86)\end{array}$ & \\
\hline & Consciência (6) & $\begin{array}{l}\text { Indica a desatenção e falta de consciência } \\
\text { das próprias respostas emocionais }(0,74)\end{array}$ & \\
\hline & Impulsos (6) & $\begin{array}{c}\text { Dificuldades de permanecer no controle } \\
\text { de um comportamento quando } \\
\text { experimenta emoções negativas. }(0,80)\end{array}$ & \\
\hline & Objetivos (5) & $\begin{array}{c}\text { Dificuldades em realizar e se concentrar } \\
\text { em tarefas ao experimentar } \\
\text { emoções negativas. }(0,85)\end{array}$ & \\
\hline & Clareza (5) & $\begin{array}{c}\text { Clareza que os indivíduos possuem } \\
\text { acerca das emoções que estão } \\
\text { experimentando. }(0,75)\end{array}$ & \\
\hline \multirow{2}{*}{ ERQ-Brasil } & $\begin{array}{l}\text { Supressão } \\
\text { emocional (4) }\end{array}$ & $\begin{array}{l}\text { Não expressão de algumas emoções } \\
\text { para ter o controle emocional. }(0,69)\end{array}$ & \multirow{2}{*}{$\begin{array}{l}\text { Escala Likert de } 7 \text { pontos } \\
\text { (1="Discordo totalmente" e } \\
7=\text { "Concordo plenamente") }\end{array}$} \\
\hline & $\begin{array}{l}\text { Reavaliação } \\
\text { emocional (6) }\end{array}$ & $\begin{array}{l}\text { Modificar os pensamentos diante da situação } \\
\text { vivenciada para controlar as emoções. }(0,75)\end{array}$ & \\
\hline \multirow{2}{*}{ ERQ-Espanha } & $\begin{array}{l}\text { Supressão } \\
\text { emocional (4) }\end{array}$ & Idem à definição do ERQ-Brasil. $(0,63)$ & \multirow{2}{*}{$\begin{array}{l}\text { Idem ao } \\
\text { ERQ-Brasil }\end{array}$} \\
\hline & $\begin{array}{l}\text { Reavaliação } \\
\text { emocional (6) }\end{array}$ & Idem à definição do ERQ-Brasil. & \\
\hline \multirow{2}{*}{ ERQ-CA } & $\begin{array}{l}\text { Supressão } \\
\text { emocional (4) }\end{array}$ & Idem à definição do ERQ-Brasil. $(0,62)$ & \multirow{2}{*}{$\begin{array}{l}\text { Escala Likert de } 5 \text { pontos } \\
\text { (1="Discordo fortemente" e } \\
5=\text { "Concordo fortemente"). }\end{array}$} \\
\hline & $\begin{array}{l}\text { Reavaliação } \\
\text { emocional (6) }\end{array}$ & Idem à definição do ERQ-Brasil. $(0,71)$ & \\
\hline \multirow{2}{*}{$\begin{array}{l}\text { Emotion } \\
\text { Regulation } \\
\text { Checklist }\end{array}$} & $\begin{array}{c}\text { Regulação } \\
\text { Emocional (3) }\end{array}$ & $\begin{array}{c}\text { Nível de expressões emocionais } \\
\text { socialmente apropriadas. }\left(0,80 / 0,53^{*}\right)\end{array}$ & \multirow{2}{*}{$\begin{array}{l}\text { Escala Likert } \\
\text { de } 4 \text { pontos } \\
\text { (1="Nunca" } \\
\text { e } 4=\text { ="Sempre"). }\end{array}$} \\
\hline & $\begin{array}{c}\text { Labilidade/ } \\
\text { Negatividade (8) }\end{array}$ & $\begin{array}{c}\text { Reatividade e intensidade de emoções } \\
\text { negativas e mudanças de humor. }\left(0,85 / 0,81^{*}\right)\end{array}$ & \\
\hline \multirow{3}{*}{$\begin{array}{l}\text { Emotional } \\
\text { Regulation } \\
\text { Strategies Scale } \\
\text { for Adults }\end{array}$} & Tristeza & Controle de emoções negativas. $(0,58)$ & \multirow{3}{*}{$\begin{array}{c}\text { Escala Likert } \\
\text { de } 4 \text { pontos } \\
\text { (1="Não tem nada } \\
\text { a ver comigo" e } \\
\text { 4="Descreve-me } \\
\text { muito bem"). }\end{array}$} \\
\hline & Raiva & $\begin{array}{c}\text { Controle emocional em situações } \\
\text { que causam raiva. }(0,69)\end{array}$ & \\
\hline & Felicidade & $\begin{array}{c}\text { Controle de emoções } \\
\text { positivas. }(0,61)\end{array}$ & \\
\hline \multirow{2}{*}{ EWRS** } & $\begin{array}{l}\text { Emoções } \\
\text { positivas (4) }\end{array}$ & $\begin{array}{l}\text { Extensão com que se exige } \\
\text { cotidianamente que o trabalhador } \\
\text { expresse emoções positivas. }(0,78)\end{array}$ & \multirow{2}{*}{$\begin{array}{l}\text { Escala Likert } \\
\text { de } 5 \text { pontos } \\
\text { (1=“Nunca"e } \\
5=\text { ="Sempre"). }\end{array}$} \\
\hline & $\begin{array}{l}\text { Emoções } \\
\text { negativas (3) }\end{array}$ & $\begin{array}{c}\text { Extensão com que se exige } \\
\text { cotidianamente que o trabalhador } \\
\text { oculte emoções positivas. }(0,77)\end{array}$ & \\
\hline \multirow{3}{*}{ ELS $^{* *}$} & $\begin{array}{l}\text { Atenção } \\
\text { profunda (2) }\end{array}$ & $\begin{array}{l}\text { Atenção intencional e consciente } \\
\text { dos sentimentos para expressar } \\
\text { emoções congruentes com } \\
\text { as demandas do trabalho. }(0,69)\end{array}$ & \multirow{3}{*}{ Idem ao EWRS. } \\
\hline & $\begin{array}{c}\text { Atenção } \\
\text { superficial (2) }\end{array}$ & $\begin{array}{l}\text { Regulação das expressões emocionais, } \\
\text { sem modificar a experiência anterior. }(0,70)\end{array}$ & \\
\hline & $\begin{array}{c}\text { Variedade e } \\
\text { intensidade (3) }\end{array}$ & $\begin{array}{c}\text { Frequência e intensidade } \\
\text { das reações emocionais. }(0,80)\end{array}$ & \\
\hline $\begin{array}{l}\text { ELS (subescala } \\
\text { de regulação } \\
\text { emocional) }\end{array}$ & $\begin{array}{l}\text { Regulação } \\
\text { emocional (4) }\end{array}$ & $\begin{array}{l}\text { Capacidade de regulação de emoções } \\
\text { superficiais e profundas. }(0,79)\end{array}$ & Idem ao EWRS. \\
\hline
\end{tabular}


Tabela 2 (continuação)

Instrumentos Encontrados para Avaliar a Regulação das Emoções

\begin{tabular}{|c|c|c|c|}
\hline Instrumentos & $\begin{array}{c}\text { Nomenclatura das } \\
\text { dimensões/fatores } \\
\left(n^{\circ} \text { de itens }\right)\end{array}$ & Definição das dimensões/fatores $(\alpha)$ & Forma de Resposta \\
\hline \multirow[b]{2}{*}{$\begin{array}{l}\text { Perfil de } \\
\text { Regulação } \\
\text { Emocional }\end{array}$} & $\begin{array}{c}\text { Down Regulation } \\
\text { - Emoções } \\
\text { negativas (3) }\end{array}$ & $\begin{array}{l}\text { Cenários que estimula raiva, } \\
\text { tristeza e medo. }(0,67)\end{array}$ & \multirow{2}{*}{$\begin{array}{c}\text { O instrumento é } \\
\text { composto por seis } \\
\text { cenários, sendo } 3 \\
\text { positivos e } 3 \text { negativos, } \\
\text { os quais preveem a } \\
\text { ativação de emoções } \\
\text { positivas ou negativas. } \\
\text { Após a descrição de cada } \\
\text { situação apresentam- } \\
\text { se oito opções de } \\
\text { respostas para lidar com } \\
\text { a emoção desencadeada. } \\
\text { Tais respostas podem } \\
\text { ser adaptativas ou } \\
\text { desadaptativas. }\end{array}$} \\
\hline & $\begin{array}{l}\text { Up Regulation } \\
\text {-Emoções } \\
\text { positivas (3) }\end{array}$ & $\begin{array}{l}\text { Cenários que estimulam alegria, } \\
\text { gratidão e excitação. }(0,73)\end{array}$ & \\
\hline
\end{tabular}

Nota. ${ }^{*}$ Grupos de risco e não risco, respectivamente; ${ }^{* *}$ Instrumentos adaptados para policiais militares

Quanto às amostras das pesquisas, analisou-se se eram compostas por crianças, adolescentes, adultos ou idosos. Os resultados indicaram que 57,89\% dos estudos $(n=11)$ foram compostos por amostras de adultos, $10,53 \%$ de crianças $(n=2), 10,53 \%$ de adolescentes $(n=2)$ e de idosos $(n=1 ; 5,26 \%)$. Também foram encontradas pesquisas com amostras compostas por adolescentes e adultos $(5,26 \% ; n=1)$, crianças e adultos $(5,26 \% ; n=1)$, além de um estudo com amostras de adultos e idosos $(5,26 \% ; n=1)$.

\section{Discussão}

O objetivo do presente estudo foi analisar a produção científica referente aos instrumentos que avaliam a autorregulação emocional por meio de uma revisão integrativa de literatura na base de dados brasileira SciELO. Os resultados apresentados demonstraram proximidade no número de publicações conforme o ano, sendo a maior parte realizada nos anos de 2016 e 2014, seguidos do período entre 2011 e 2017. Santana e Gondim (2016) ressaltam que os estudos e o interesse de pesquisadores acerca da regulação emocional têm aumentado na última década, sobretudo, segundo os autores, por ser compreendida como um aspecto fundamental no processo de adaptação frente às dificuldades da vida e por trazer consequências positivas em termos afetivos e de bem-estar. Em relação aos países nos quais as publicações ocorreram, o Brasil figurou como o principal, o que não causa estranheza, tendo em vista que a base consultada é nacional.

A inteligência emocional foi o construto mais pesquisado nos artigos analisados, corroborando a forte associação com a regulação das emoções (Ruiz et al., 2012; Salovey et al., 1995). Os estudos relacionados à inteligência emocional baseavam-se na concepção de que a autorregulação emocional é um componente da inteligência emocional (Salovey et al., 1995), apresentavam correlações de magnitudes altas (Espinoza-Venegas et al., 2015; Ruiz et al., 2012; Teques et al., 2015), utilizavam a teoria de desenvolvimento da TMMS-24, a qual considera o monitoramento, avaliação e regulação de sentimentos e emoções, e possuíam algum fator que avaliava a regulação emocional. Identificar, nomear e agir adequadamente em relação aos próprios sentimentos e aos de outra pessoa são características que definem uma pessoa com altos níveis de inteligência emocional (Goleman, 2006). Todavia, antes de considerar a inteligência emocional de forma ampla, parece ser necessário dar um foco maior aos níveis de autoerregulação emocional de um indivíduo em um processo avaliativo, posto que são encontrados baixos níveis de autorregulação emocional em pessoas com maiores dificuldades de estabelecer relações sociais (Gratz \& Roemer, 2004; Weiss, Gratz, \& Lavender, 2015), fato que pode indicar dificuldades na compreensão dos sentimentos e emoções alheios. Ademais, tais resultados podem justificar a escolha de um instrumento que avalia a inteligência emocional para identificar aspectos da regulação emocional (Espinoza-Venegas et al., 2015; Espinoza-Venegas \& Sanhueza-Alvarado, 2012; IsazaZapata \& Calle-Piedrahita, 2016; Pavarini, Loureiro, \& Souza, 2011; Teques et al., 2015).

Em outra medida, a depressão e a ansiedade são construtos que podem indicar dificuldades psiquiátricas e também foram associadas à regulação das emoções nas pesquisas analisadas. Os estudos encontrados nessa temática faziam comparações entre grupos com e sem diagnósticos, como câncer, ansiedade e depressão (Coutinho, Ribeirinho, Ferreirinha, \& Dias, 2010; Porro et al, 2012). Nesses estudos, foi identificado que os grupos com diagnósticos podem apresentar maiores dificuldades ao expressarem suas emoções, se 
comparados aos grupos sem diagnósticos, além de fazerem mau uso das emoções negativas, sendo importante o desenvolvimento de programas que auxiliem os indivíduos na prevenção da depressão e da ansiedade em grupos clínicos. Os achados auxiliam a compreender a quantidade de estudos que objetivaram desenvolver programas de intervenção e também aqueles que se dedicaram à construção e validação instrumentos de autorregulação emocional, uma vez que ela pode minimizar a ansiedade e a depressão, além de auxiliar em comportamentos adaptativos em relacionamentos interpessoais (Gratz \& Roemer, 2004; Nelis et al., 2011; Weiss et al., 2015).

Nos trabalhados analisados, foi possível identificar o uso de dois modelos conceituais mais frequentes em relação à regulação das emoções: o modelo adotado no Emotional Regulation Questionnaire (ERQ) (Gratz \& Roemer, 2004; Gross \& John, 2003) e o da Difficulties in Emotion Regulation Scale (DERS), o que em certa medida era esperado, dada à adesão a tais modelos em diversos países. O modelo conceitual que embasou a DERS possibilitou o desenvolvimento de dois instrumentos, sendo que um investiga o controle da emoção negativa (DERS-Negative) e o outro o controle da emoção positiva (DERS-Positive) (Gratz \& Roemer, 2004; Weiss et al., 2015). A diferença do modelo da DERS e o da ERQ é que este último possui dois fatores que se aplicam tanto a emoções negativas quanto positivas, enquanto a DERS possui um instrumento para avaliar emoções positivas e outro para emoções negativas (Gratz \& Roemer, 2004; Gross \& John, 2003; Weiss et al., 2015), o que parece indicar que a ERQ é um instrumento mais geral e a DERS mais específico.

No que se refere ao uso de escalas que avaliam as emoções no contexto organizacional e do trabalho, foram utilizadas a ELS e a EWRS. Cabe ressaltar a importância da investigação no contexto do trabalho, pois a depender das atividades desempenhadas, como o contato com clientes e pressão para alcançar metas, o funcionário pode desenvolver um desgaste emocional, estresse e Burnout (Rodrigues, Carlotto, \& Câmara, 2017). Rodrigues et al. (2017) encontraram uma associação negativa entre o esforço para regular as emoções no trabalho com sentimentos de culpa, indolência, ilusão pelo trabalho e desgaste emocional.

Ademais, quanto aos instrumentos compostos por pranchas e cenários para identificar a regulação emocional, ressalta-se a importância de tais achados. As imagens, os questionários e a condução do avaliador em conjunto permitem que mais recursos sejam utilizados para avaliar o construto, além de estimular a vivência e identificação de emoções em casos de maiores complexidades, como amostras clínicas e/ou infantis, ou conforme a variação dos diversos contextos nos quais as pessoas estão inseridas (Cruvinel \& Boruchovitch, 2011; Gondim et al., 2015; Nelis et al., 2011).

\section{Considerações Finais}

O intuito do presente estudo, analisar a produção científica relacionada aos instrumentos de autorregulação emocional em uma base de dados brasileira no período entre 2008 e 2017 por meio de uma revisão de literatura, indicou resultados satisfatórios para o desenvolvimento de novos estudos. Os artigos analisados demonstraram que existem divergências conceituais em relação à autorregulação emocional, todavia, os modelos de Gross e John (2003) e Gratz e Roemer (2004) parecem ser os mais aceitos pelos pesquisadores, sobretudo no desenvolvimento de instrumentos, posto que já foram realizados estudos de validação com as bases teóricas desses instrumentos em diversos países, como a China, Itália, Colômbia, Chile, Portugal e Turquia.

O crescimento gradual de pesquisas relacionadas ao tema e os tipos de amostras e contextos em que essas amostras estavam inseridas revelam que regular as próprias emoções é um importe recurso para preservar a saúde mental (diminuição de estados depressivos e de ansiedade) e física (controle na ingestão de alimentos e perda de peso). Novas áreas podem ser investigadas como forma de compreender a autorregulação emocional dos indivíduos diante das adversidades. $\mathrm{Na}$ área esportiva, por exemplo, as investigações poderiam ser focadas no enfrentamento que atletas possuem diante de situações estressantes, como os treinamentos excessivos, controle na alimentação e derrotas. Além disso, considerar como os atletas controlam as emoções nas vitórias, diante da fama e do êxito, uma vez que existem diferenças relacionadas ao controle das emoções positivas e negativas.

$\mathrm{Na}$ área organizacional, compreender o controle das emoções parece ser uma forma de contribuir com o desenvolvimento de empresas, trabalhadores e empregadores, uma vez que as relações possivelmente se tornam mais harmoniosas e saudáveis em indivíduos que conseguem se controlar emocionalmente. Ainda, a autorregulação emocional pode ser investigada no contexto hospitalar como um recurso para minimizar os processos estressantes para familiares, pacientes e profissionais. $\mathrm{O}$ desenvolvimento de grupos de sala de espera, por exemplo, com foco na aprendizagem e entendimento das próprias emoções para pessoas envolvidas na dinâmica hospitalar seria uma forma de ampliar o conhecimento acerca do construto.

Destaca-se que o presente estudo buscou analisar a autorregulação por meio de apenas uma base de dados brasileira e somente com descritores em português, o que pode ser uma limitação. Foi identificado que os estudos no Brasil sobre a temática são incipientes, o que permite pouca generalização dos resultados em termos mundiais. Faz-se importante o desenvolvimento e/ou adaptação de outras medidas que avaliem o construto no cenário brasileiro, sobretudo pelo fato das diferenças 
culturais de cada país interferirem na construção e no processo avaliativo de cada instrumento, como observado na presente revisão. Como agenda de pesquisa, parece ser indispensável a mesma análise, utilizando-se outras bases e repositórios que permitam a compreensão mais ampla da pesquisa do construto.

\section{Referências}

Amorim Neto, R. C., \& True, M. (2011). The development and treatment of impulsivity. Psico, 42(1), 134-141. Recuperado de http:// revistaseletronicas.pucrs.br/ojs/index.php/revistapsico/article/viewFile/7665/6309

Arcoverde, R. L., \& Soares, L. S. L. C. (2012). Funções neuropsicológicas associadas a condutas autolesivas: Revisão integrativa de literatura. Psicologia: Reflexão e Crítica, 25(2), 293-300. doi: 10.1590/S0102-79722012000200011

Barros, L., Goes, A. R., \& Pereira, A. I. (2015). Parental self-regulation, emotional regulation and temperament: Implications for intervention. Estudos de Psicologia, 32(2), 295-306. doi: 10.1590/0103-166X2015000200013

Berking, M., Wirtz, C. M., Svaldi, J., \& Hofmann, S. G. (2014). Emotion regulation predicts symptoms of depression over five years. Behavior Research and Therapy, 57(1), 13-20. doi: 10.1016/j.brat.2014.03.003

Best, R. G., Downey, R. G, \& Jones, R. G. (1997, Abril). Incumbent perceptions of emotional work requirements. Em K. Willians (Org.), Paper presented at the 12th anual meeting of the Society for Industrial and Organizational Psychology, Missouri, EUA.

Brotheridge, C. M., \& Lee, R. (2003). Development and validation of the emotional labor scale. Journal of Ocupational and Organizational Psychology, 76(3), 363-379. doi: 10.1348/096317903769647229

Bueno, J. M. H. (2008). Construção de um Instrumento para Avaliação da Inteligência Emocional em Crianças (Tese de doutorado), Universidade São Francisco, Campinas, SP. Recuperado de https://www.usf.edu.br/galeria/getImage/427/6932606611633978.pdf

Cabello, R., José M., S., Fernández-Berrocal, P., \& Gross, J. J. (2012). A spanish adaptation of the emotion regulation questionnaire. European Journal of Psychological Assessment, 29(2013), 234-240. doi: 10.1027/1015-5759/a000150

Castillo, J. A. G., Dias., P. C., \& Castelar-Perim, P. (2012). Autorregulação e consumo de substâncias na adolescência. Psicologia: Reflexão e Crítica, 25(2), 238-247. doi: 10.1590/S0102-79722012000200005

Coutinho, J., Ribeiro, E., Ferreirinha, R., \& Dias, P. (2010). Versão portuguesa da Esca de Dificuldades de Regulação Emocional e sua relação com Sintomas Psicopatológicos. Revista de Psiquiatria Clínica, 37(4), 145-151. doi: 10.1590/S0101-60832010000400001

Cruvinel, M., \& Boruchovitch, E. (2011). Regulação emocional em crianças com e sem sintomas de depressão. Estudos de Psicologia, 16(3), 219-226. doi: 10.1590/S1413-294X2011000300003

Espinoza-Venegas, M., \& Sanhueza-Alvarado., O. (2012). Miedo a la muerte y su relación con la inteligencia emocional de estudiantes de enfermería de Concepción. Acta Paulista de Enfermagem, 25(4), 607-613. doi: 10.1590/S0103-21002012000400020

Espinoza-Venegas, M., Sanhueza-Alvarado, O., Ramírez-Elizondo, N., \& Sáez-Carrillo, K. (2015). Validação do construto e da confiabilidade de uma escala de inteligência emocional aplicada a estudantes de enfermagem. Revista Latino-Americana de Enfermagem, 23(1), $139-147$. doi: 10.1590/0104-1169.3498.2535

Gaspar, T., \& Matos, M. G. (2015). "Para mim é fácil”: Escala de avaliação de competências pessoais e sociais. Psicologia, Saúde E Doenças, 16(2), 195-206. doi: 10.15309/15psd160206

Gaspar, T., Tomé, G. Q., Simões. C., \& Matos, M. G. (2015). Estratégias de auto-regulação em pré-adolescentes e adolescentes: Versão portuguesa do TESQ-E. Psicologia: Reflexão e Crítica, 28(4), 649-658. doi: 10.1590/1678-7153.201528402

Goleman, D. (2006). Inteligência Social - A nova ciência das relações humanas. Barcelos: Círculo de Leitores.

Gondim, S. M. G., \& Borges-Andrade, J. E. (2009). Regulação emocional no trabalho: Um estudo de caso após desastre aéreo. Psicologia: Ciência e Profissão, 29(3), 512-533. doi: 10.1590/S1414-98932009000300007

Gondim, S. M. G., Pereira, C. R., Hirschle, A. L. T., Palma, E. M. S., Alberton, G. D., Paranhos, J., ... Ribeiro, W. B. R (2015). Evidências de validação de uma medida de características pessoais de regulação das emoções. Psicologia: Reflexão e Crítica, 28(4), 659-667. doi: 10.1590/1678-7153.201528403

Granada, X. O., Andreu, M. G., \& Guiu, G. F. (2014). Regulación emocional de los resultados adversos en competición. Estrategias funcionales en deportes colectivos. Cuadernos de Psicología del Esporte, 14(1), 63-72. Recuperado de http://scielo.isciii.es/scielo.php?script=sci_artte $\mathrm{xt} \&$ pid $=$ S1578-84232014000100008

Gratz, K. L., \& Roemer, L. (2004). Multidimensional assessment of emotion regulation and dysregulation: Development factor structure, and initial validation of the dificulties in emotion regulation scale. Journal of Psychopatology and Behavioral Assessment, 26(1), 41-54. doi: 10.1007/s10862-008-9102-4

Gross, J. J., \& John, O. P. (2003). Individual differences in two emotion regulation processes: Implications for affect, relationships, and wellbeing. Journal of Personality and Social Psychology, 85(2), 348-362. doi: 10.1037/0022-3514.85.2.348

Gullone, E., \& Taffe, J. (2012). The emotion regulation questionnaire for children na adolescents (ERQ-CA): A psychometric evaluation. Psychology Assessment, 24(2), 409-417. doi: 10.1037/a0025777

Isaza-Zapata, G. M., \& Calle-Piedrahita, J. S. (2016). Um acercamiento a la comprensión del perfil de la Inteligencia Emocional. Revista Latinoamericana de Ciencias Sociales, Nyñes y Juventud, 14(1), 331-345. doi: 10.11600/1692715x.14122220814

Lopes, R. M. F., Nascimento, R. F. L., \& Bandeira, D. R. (2005). Avaliação do transtorno de déficit de atenção/hiperatividade em adultos (TDAH): Uma revisão de literatura. Avaliação Psicológica, 4(1), 65-74. Recuperado de http://pepsic.bvsalud.org/scielo.php?script=sci_ arttext\&pid=S1677-04712005000100008

McKnown, C., Gumbiner, L. M., Russo, N. M., \& Lipton, M. (2009). Social-emotional learning skill, self-regulation, and social competence in typically developing and clinic-referred children. Journal of Clinical Child \& Adolescence Psychology, 38(6), 858-871. doi: 10.1080/15374410903258934

Muñoz-Martínez, A. M., Vargas, R. M., \& Hoyos-González, J. S. (2016). Escala de Dificultades en Regulación Emocional (DERS): Análisis factorial en una muestra colombiana. Acta Colombiana de Psicologia, 19(1), 225-236. doi: 10.14718/ACP.2016.19.1.10 
Nelis, D., Quoidbach, J., Hansenne, M., \& Mikolajczak, M. (2011). Measuring individual differences in emotion regulation: The Emotion Regulation Profile-Revised (ERP-R). Psychologica Belgica, 51(1), 49-91. doi:10.5334/pb-51-1-49

Pavarini, G., Loureiro, C. P., \& Souza, D. H. (2011). Compreensão de emoções, aceitação social e avaliação de atributos comportamentais em crianças escolares. Psicologia: Reflexão e Crítica, 24(1), 135-143. doi: 10.1590/S0102-79722011000100016

Pinto, H. M., Carvalho, A. R., \& Sá, E. N. (2014). Os estilos educativos parentais e a regulação emocional: Estratégias de regulação e elaboração emocional das crianças em idade escolar. Análise Psicológica, 32(4), 387-400. doi: 1014417/ap.32.3.844

Porro, M. L., Andrés, M. L., \& Rodríguez-Espínola, S. (2012). Regulación emocional y cáncer: Utilización diferencial de la expresión y supresión emocional en pacientes oncológicos. Avances en Psicología Latinoamericana, 30(2), 341-355. Recuperado de http://www.scielo. org.co/scielo.php?script $=$ sci_arttext\&pid =S1794-47242012000200010\&lang $=$ pt

Rodrigues, S. Y. S., Carlotto, M. S., \& Câmara, S. G. (2017). Impacto da regulação de emoções no trabalho sobre as dimensões de Burnout em psicólogos: O papel moderador da autoficácia. Análise Psicológica, 35(2), 191-201. doi: 10.14417/ap.1147

Ruiz, E., Salazar, I. C., \& Caballo, V. E. (2012). Inteligencia emocional, regulação emocional y estilos/transtornos de personalidad. Behavioral Psychology/ Psicologia Conductal, 20(2), 281-304. Recuperado de https://www.researchgate.net/profile/Vicente_Caballo/ publication/230704262_Emotional_intelligence_emotional_regulation_and_personality_stylesdisorders_Inteligencia_emocional_ regulacion_emocional_y_estilostrastornos_de_personalidad/links/0046352c1bf9fc595d000000.pdf

Salovey, P., Mayer, J. D., Goldman, S. L., Turvey, C., \& Palfai, T. P. (1995). Emotional attention, clarity and repair: Exploring emotional intelligence using the Trait Meta-Mood Scale. Em J. W. Pennebaker (Ed.), Emotion disclosure and health (pp. 125-154). Washington, DC: APA Books.

Santana, V. S., \& Gondim, S. M. G. (2016). Regulação emocional, bem-estar psicológico e bem-estar subjetivo. Estudos de Psicologia, 21(1), 58-68. doi: 10.5935/1678-4669.20160007

Schlindwein-Zanini, R. (2010). Demência no idoso: Aspectos neurológicos. Revista de Neurociências, 18(2), 220-226. Recuperado de http:// revistaneurociencias.com.br/edicoes/2010/RN1802/262\%20revisao.pdf

Sheppes, G., Scheibe, S., Suri, G., \& Gross, J. J. (2011). Emotion Regulation Choice. Psychological Science, 22(11), 1391-1396. doi: $10.1177 / 0956797611418350$

Silva, I. (2015). Importance of emotional regulation in obesity and weight loss treatment. Fractal: Revista de Psicologia, 27(3), 286-290. doi: 10.1590/1984-0292/1503

Teques, A. P., Llorca-Ramón, G., Bueno-Carrera, G., Pais-Ribeiro, J., \& Teques, P. (2015). Desenvolvimento e avaliação das características psicométricas do Questionário de Auto-Percepção de Inteligência Emocional (QIE-AP). Psicologia: Reflexão e Crítica, 28(2), $270-279$. doi: 10.1590/1678-7153.201528207

Tharp, A. A., Schumacher, J. A., McLeish, A. C., Samper, R. E., \& Coffey, S. F. (2012). Relative importance of emotional dysregulation, hostility, and impulsiveness in predicting intimate partner violence perpetrated by men in alcohol treatment. Psychology of Women Quarterly, 37(1), 51-60. doi: 10.1177/0361684312461138

Weiss, N. H., Gratz, K. L., \& Lavender, J. M. (2015). Factor structure and initial validation of a multidimensional measure of difficulties in the regulation of positive emotions: The DERS-Positive. Behavior Modification, 39(3), 431-453. doi:10.1177/0145445514566504

\section{Sobre os autores}

Helder Henrique Viana Batista é psicólogo, mestre e doutorando pela Universidade São Francisco, atualmente auxilia no projeto de "Construção de Instrumento de Avaliação de Autorregulação e Estudos Psicométricos" e no projeto "Estudos para Construção e Validação de Instrumentos de Avaliação das Forças de Caráter".

Ana Paula Porto Noronha é psicóloga, mestre, doutora pela Pontifícia Universidade Católica de Campinas, professora do Programa de Pós-Graduação Stricto Sensu da Universidade São Francisco, coordenadora dos projetos "Construção de Instrumento de Avaliação de Autorregulação e Estudos Psicométricos” e "Estudos para Construção e Validação de Instrumentos de Avaliação das Forças de Caráter” e recebe financiamento de pesquisa do CNPq. 